\title{
Increased NY-ESO-1 Expression and Reduced Infiltrating CD3+ T Cells in Cutaneous Melanoma
}

\author{
Mara Giavina-Bianchi, ${ }^{1}$ Pedro Giavina-Bianchi, ${ }^{2}$ Mirian Nacagami Sotto, ${ }^{1}$ \\ Alona Muzikansky, ${ }^{3}$ Jorge Kalil, ${ }^{2}$ Cyro Festa-Neto, ${ }^{1}$ and Lyn M. Duncan ${ }^{4}$ \\ ${ }^{1}$ Department of Dermatology, University of São Paulo, Avenida Dr. Enéas de Carvalho Aguiar 255, $3^{\circ}$ Andar, \\ 05403-900 São Paulo, SP, Brazil \\ ${ }^{2}$ Division of Clinical Immunology and Allergy, University of São Paulo, Avenida Dr. Enéas de Carvalho Aguiar 255, \\ $8^{\circ}$ Andar, 05403-900 São Paulo, SP, Brazil \\ ${ }^{3}$ MGH Biostatistics Center, 50 Staniford Street, Suite 560, Boston, MA 02114, USA \\ ${ }^{4}$ Dermatopathology Unit, Pathology Service, Massachusetts General Hospital, Harvard Medical School, Warren Building 825, \\ 55 Fruit Street, Boston, MA 02114, USA
}

Correspondence should be addressed to Mara Giavina-Bianchi; clinicadermato@ig.com.br

Received 5 February 2015; Revised 23 March 2015; Accepted 29 March 2015

Academic Editor: Shigeo Koido

Copyright (C) 2015 Mara Giavina-Bianchi et al. This is an open access article distributed under the Creative Commons Attribution License, which permits unrestricted use, distribution, and reproduction in any medium, provided the original work is properly cited.

\begin{abstract}
NY-ESO-1 is a cancer-testis antigen aberrantly expressed in melanomas, which may serve as a robust and specific target in immunotherapy. NY-ESO-1 antigen expression, tumor features, and the immune profile of tumor infiltrating lymphocytes were assessed in primary cutaneous melanoma. NY-ESO-1 protein was detected in 20\% of invasive melanomas (16/79), rarely in in situ melanoma (1/10) and not in benign nevi (0/20). Marked intratumoral heterogeneity of NY-ESO-1 protein expression was observed. NY-ESO-1 expression was associated with increased primary tumor thickness $(P=0.007)$ and inversely correlated with superficial spreading melanoma $(P<0.02)$. NY-ESO-1 expression was also associated with reduced numbers and density of CD3+ tumor infiltrating lymphocytes $(P=0.017)$. When NY-ESO-1 protein was expressed, CD3+ T cells were less diffusely infiltrating the tumor and were more often arranged in small clusters $(P=0.010)$ or as isolated cells $(P=0.002)$ than in large clusters of more than five lymphocytes. No correlation of NY-ESO-1 expression with gender, age, tumor site, ulceration, lymph node sentinel status, or survival was observed. NY-ESO-1 expression in melanoma was associated with tumor progression, including increased tumor thickness, and with reduced tumor infiltrating lymphocytes.
\end{abstract}

\section{Introduction}

Cutaneous melanoma is a global health problem, with increasing incidence in Caucasians [1-4]. While more than $95 \%$ of patients with early stage melanoma are recurrencefree 5 years after diagnosis, fewer than $50 \%$ of patients with metastases survive more than 5 years [5]. Despite the revolutionary developments in immunotherapy, the treatment of patients with metastatic melanoma has been focused more on extending progression free survival and palliation than on effecting a cure [6-10].

Consistent with the recent successes of immunotherapy, there is interest in vaccine development for melanoma. Cancer-testis antigens, such as NY-ESO-1, may serve as robust and specific targets; while they are normally expressed only in the germ cells of the adult testis, they are aberrantly expressed in tumors [11-17].

NY-ESO-1 is a well-characterized cancer-testis antigen expressed in $20-30 \%$ of carcinomas (lung, esophagus, liver, stomach, prostate, ovary, endometrium, and bladder) and some sarcomas [18-27]. Prior reports showed NY-ESO-1 expression in 20 to $40 \%$ of melanomas; metastases more likely express this antigen than primary cutaneous melanoma [2831]. NY-ESO-1 is a highly immunogenic antigen in vitro and is presented to T cells via HLA. The presence of circulating NY-ESO-1 antigen-specific T cells in patients with metastatic melanoma correlates with better prognosis $[32,33]$. 
To further understand the role of NY-ESO-1 in melanoma progression, we studied the expression of NY-ESO-1 protein in tissue sections of benign and malignant cutaneous melanocytic tumors and analyzed the association if any with melanoma progression and melanoma-specific survival. We also assessed the immune profile of the tumor infiltrating lymphocytes (TIL) in primary invasive melanoma and in the context of NY-ESO-1 antigen expression.

\section{Materials and Methods}

2.1. Case Selection. This cohort was composed of 109 Brazilian patients with melanocytic skin tumors: benign nevi $(n=$ $20)$, melanoma in situ $(n=10)$, and invasive primary cutaneous melanoma $(n=79)$. Data was collected from the Division of Dermatology, University of São Paulo, and this study has been approved by the Institutional Review Boards of University of São Paulo (CAPPesq) and of Massachusetts General Hospital (2013P000172).

Demographic features assessed included gender, age, skin phototype, tumor site, sentinel lymph node status, development of metastasis, and melanoma-associated death. Histopathological evaluation included tumor thickness, histological type, ulceration, and grade of tumor infiltrating lymphocytes (TIL). TIL were graded as brisk when lymphocytes diffusely infiltrated throughout the tumor or were present as a broad band at the advancing tumor margin; nonbrisk when there was focal or multifocal infiltration; and absent when there were no lymphocytes infiltrating the tumor or the lymphocytic infiltrate was not associated with the tumor cells [34].

2.2. Immunohistochemistry for NY-ESO-1, CD3, CD8, FoxP3, and CD8FoxP3 Dual Staining. Immunohistochemical detection of NY-ESO-1 was performed on formalin-fixed paraffinembedded tissue sections with a monoclonal antibody (E978, Ludwig Institute, Brazil), antigen retrieval with tris-EDTA buffer $(\mathrm{pH}=9.0)$, DAB chromogen (Biocare, California, USA), and hematoxylin counterstain [29]. Normal testis served as a positive control. NY-ESO-1 expression was scored microscopically and independently by three of the authors (Mara Giavina-Bianchi, Mirian Nacagami Sotto, and Lyn M. Duncan). Samples were scored for (1) intensity (0: negative; 1+: weak, 2+: moderate, and 3+: intense); (2) distribution (complete, regional, or scattered); and (3) percentage of tumor cell staining. Cases staining with intensity of 0 or $1+$ (negative or weak) and/or presenting less than $2 \%$ of the tumor cells positive were considered to be negative. Tumors that displayed staining with intensity 2 or $3+$ (moderate or strong) and presenting more than $2 \%$ of the tissue cells staining for the antigen were considered positive (Figures 1(a), 1(b), 1(c), and 1(d)).

Immunohistochemical detection of CD3 was performed similarly (LN10, Leica Bond Polymer Refined Detection Kit Cat. number DS9800 and Bond Polymer Red Detection Kit Cat. number DS9390, Illinois, USA); CD8 and FoxP3 double stains utilized antibodies SP16 (Biocare Medical, California,
USA) and FJK-16s (eBioscience, California, USA), respectively, detected with red permanent chromogen (DAKO, California, USA) for anti-CD8 and blue substrate (Vector Labs, California, USA) for anti-FoxP3 antibodies.

2.3. Quantification of TIL. We adapted the morphometric point-counting method previously described for mast cell quantification to assess the volume density of lymphocyte subsets $[35,36]$. Briefly, slides are viewed in a microscope using a specialized eyepiece graticule that contains a $10 \times$ $10 \mathrm{~mm}$ square grid (100 contiguous squares). The intersection of any two lines defined individual points, and cells at an intersection within a 400x magnification field were counted. The grid was superimposed over the TIL stained for CD3, CD8, FoxP3, and CD8FoxP3. Whenever possible, the four fields with highest cell density, either in the tumor or at the periphery, were counted and the mean per each slide was used for statistical analysis. The distribution pattern of CD3+ T cells was reported as isolated cell (only one cell), small clusters (2 to 5), or large clusters (6 or more T cells).

2.4. Statistical Analysis. Association between NY-ESO-1 expression and tumor thickness was performed using a two tailed Student's $t$-test. Associations between NY-ESO-1 expression and gender, age, site, phototype, CD3+, and CD8+FoxP3- cells were analyzed by Pearson's chi-square test. Associations between NY-ESO-1 expression and melanoma type, ulceration, CD8-FoxP3+, and CD8+FoxP3+ cells were analyzed by Fisher's exact test. $P$ value of $\leq 0.05$ was considered to be statistically significant. Statistical analysis was performed by SAS software.

\section{Results}

The 79 patients with invasive primary melanoma ranged in age from 23 to 92 years (mean 57.8). There were 31 men (40\%) and 47 women $(60 \%)$. The predominat skin phototype was Fitzpatrick II (35/65; 56\%), and the most common tumor sites were trunk and extremity (37\% and 35\%, resp.; Table 1).

We assessed cutaneous melanomas having all tumor thickness: $\leq 1.0 \mathrm{~mm}(n=24,30 \%) ; 1.01-2.0 \mathrm{~mm}(n=23,29 \%)$; 2.01-4.0 $\mathrm{mm}(n=6,8 \%)$; and $>4.0 \mathrm{~mm}(n=26,33 \%)$. Superficial spreading melanoma (SSM) was the most frequent histological type, 39\% (31/79), followed by nodular, 33\% (26/79), acral lentiginous, $10 \%(8 / 79)$, and lentigo maligna melanoma, $9 \%(7 / 79)$. Vertical growth phase was present in $86 \%$ of the tumors (68/79) and ulceration was observed in 20\% (16/79; Table 1).

Benign melanocytic nevi ( $n=20 ; 9$ dermal nevi and 11 dysplastic nevi) and most in situ melanomas (9/10) did not express NY-ESO-1. Regarding the 79 primary cutaneous invasive melanomas, those in radial growth phase did not express NY-ESO-1 (0/11), whereas NY-ESO-1 protein was expressed by melanoma cells in $24 \%(16 / 68)$ of the tumors presenting vertical growth phase.

The mean tumor thickness of NY-ESO-1 positive melanomas was greater than NY-ESO-1 negative tumors $(5.2 \mathrm{~mm}$ \pm 1.07 versus $2.7 \mathrm{~mm} \pm 0.36 ; P=0.007$; Figure 2$)$. NY-ESO-1 


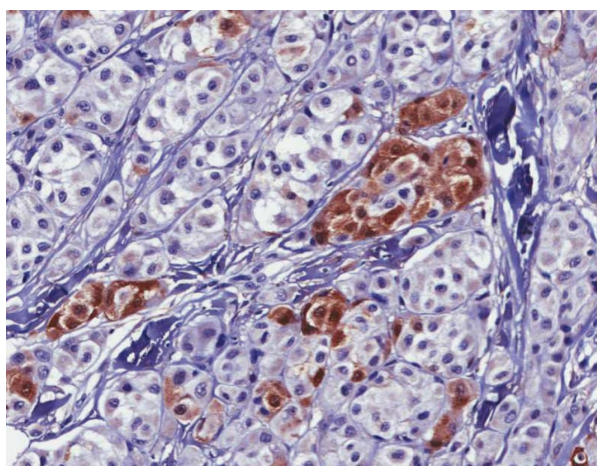

(a)

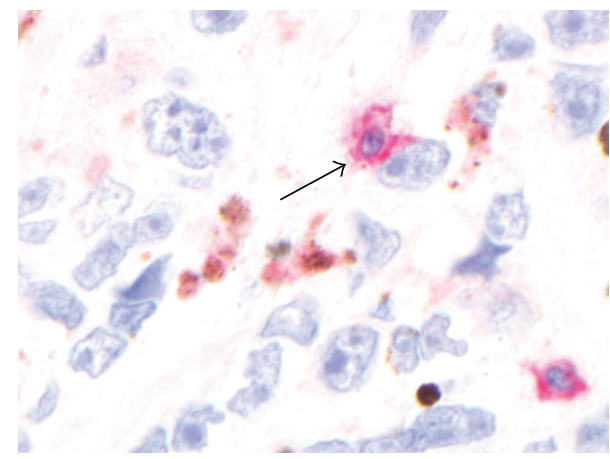

(c)

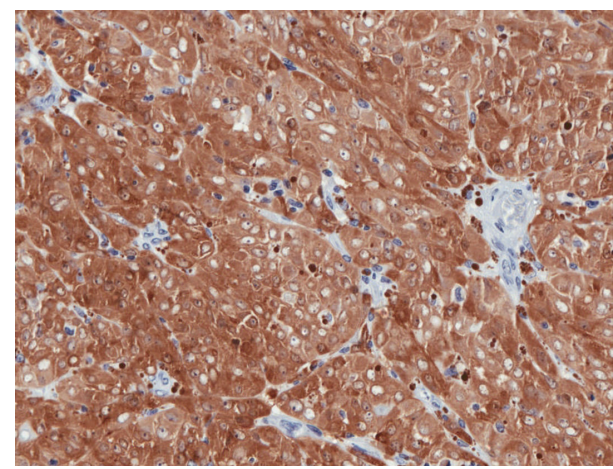

(b)

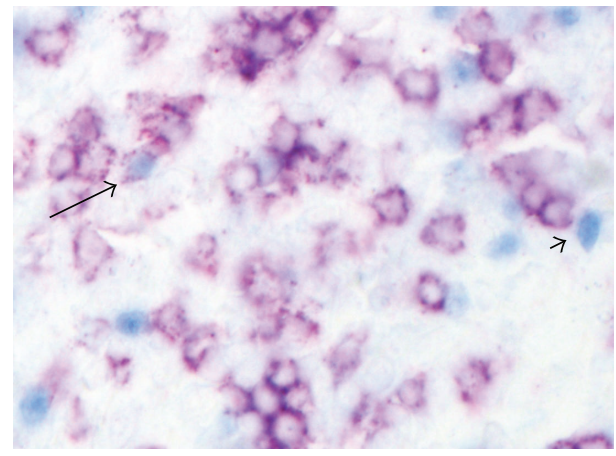

(d)

FIgURE 1: Immunohistochemical detection of NY-ESO-1 (+) tumor cells and CD3+, CD8+ FOXP3-, CD8-FOXP3+, and CD8+FOXP3+ infiltrating lymphocytes in primary cutaneous melanoma. (a) NY-ESO-1 regional expression, $3+$ intensity $(\times 400)$; (b) NY-ESO-1 complete expression, 3+ intensity $(\times 200)$; (c) CD3+ lymphocyte (red, arrow) embracing a melanoma cell $(\times 630)$; (d) CD8+FOXP3- lymphocytes (membranous purple staining), CD8-FOXP3+ lymphocytes (blue nuclear staining, arrowhead), and CD8+FOXP3+ cell (purple membranous and blue nuclear staining, arrow), $\times 400$.

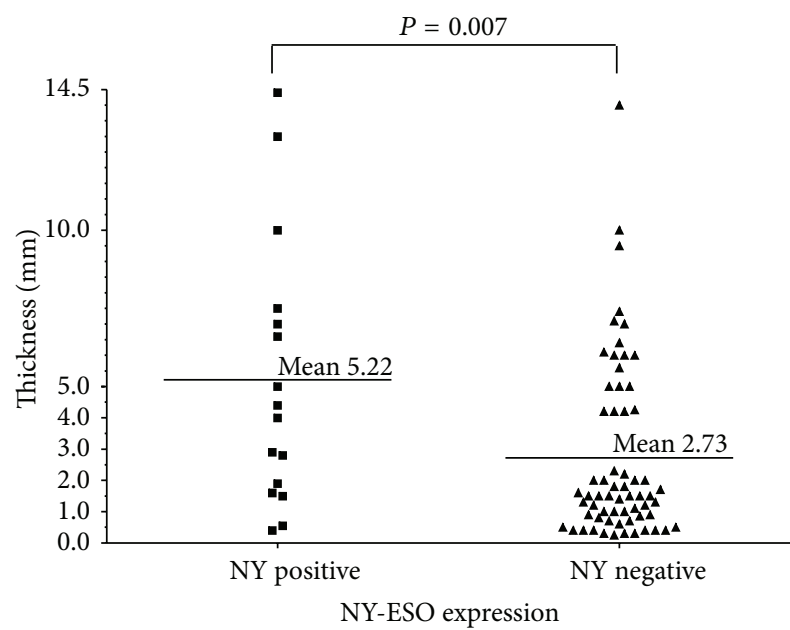

FIGURE 2: Association between primary cutaneous melanoma thickness and NY-ESO-1. Each point represents one patient, and the horizontal bars represent the mean melanoma thickness of each group $(P=0.007)$.

was expressed significantly less frequently in SSM when compared to other subtypes (Table $1, P<0.02$ ).
Sentinel lymph node (SLN) biopsy was performed in $23 \%(18 / 78)$ of patients, of which $11 \%(2 / 18)$ were positive. The criteria used to indicate sentinel lymph node biopsy at University of São Paulo were clinically localized primary cutaneous melanomas with thickness between 1.0 and $4.0 \mathrm{~mm}$. Regarding the 61 cases without sentinel lymph node biopsy, $57 \%$ (45/79) did not meet the criteria and 20\% (16/79) were diagnosed with melanoma before sentinel lymph node biopsy becomes the standard of care.

The mean follow-up was 108 months (7-264 months). No evidence of recurrent or metastatic melanoma at the last appointment date (no evidence of disease (NED)) was identified in 54\% (37/68); $21 \%(14 / 68)$ were alive with metastatic disease (alive with disease (AWD)), 9 of those 14 having 4 or more years of follow-up; $21 \%(14 / 68)$ had died because of melanoma (dead of disease (DOD)). Three patients (4\%) with less than 7-month follow-up were considered lost to followup (Table 1).

No correlation of NY-ESO-1 expression with SLN positivity, tumor recurrence, metastasis, or overall survival was detected; however, low numbers in some groups may have confounded the data. NY-ESO-1 expression was not shown to correlate with gender, age at diagnosis, tumor site, skin phototype, or ulceration (Table 1). 
TABLE 1: Clinical and histopathological parameters in NY-ESO-1 positive and negative primary cutaneous melanomas.

\begin{tabular}{|c|c|c|c|c|}
\hline Parameter & Subgroup & Number of patients (\%) & $\begin{array}{c}\text { NY-ESO-1 (+) } \\
n(\%)\end{array}$ & $\begin{array}{c}\text { NY-ESO-1 (-) } \\
n(\%)\end{array}$ \\
\hline \multirow{2}{*}{ Gender } & Male & $31(40)$ & $8(26)$ & $23(74)$ \\
\hline & Female & $47(60)$ & $8(17)$ & $39(83)$ \\
\hline \multirow{6}{*}{ Skin phototype } & I & $1(2)$ & $0(0)$ & $1(100)$ \\
\hline & II & $35(56)$ & $7(20)$ & $28(80)$ \\
\hline & III & $22(35)$ & $5(22)$ & $17(78)$ \\
\hline & IV & $4(6)$ & $2(50)$ & $2(50)$ \\
\hline & $\mathrm{V}$ & $2(3)$ & $0(0)$ & $2(100)$ \\
\hline & VI & $1(2)$ & $0(0)$ & $1(100)$ \\
\hline Median age (years) & & 78 & 55.2 & 58.3 \\
\hline \multirow{4}{*}{ Location } & Extremity & $28(35)$ & $6(21)$ & $22(79)$ \\
\hline & Trunk & $29(37)$ & $6(21)$ & $23(79)$ \\
\hline & Head and neck & $14(18)$ & $1(7)$ & $13(93)$ \\
\hline & Acral (Hand and foot) & $8(11)$ & $3(37.5)$ & $5(62.5)$ \\
\hline \multirow{5}{*}{ Melanoma subtype } & Superficial spreading & $31(39)$ & $2(6)^{*}$ & $29(94)^{*}$ \\
\hline & Nodular & $26(33)$ & $6(23)$ & $20(77)$ \\
\hline & Lentigo maligna & $7(9)$ & $3(43)$ & $4(57)$ \\
\hline & Acral lentiginous & $8(10)$ & $3(37.5)$ & $5(62.5)$ \\
\hline & Unclassified & $7(9)$ & $2(29)$ & $5(71)$ \\
\hline \multirow{2}{*}{ Ulceration } & Present & $16(20)$ & $5(31)$ & $11(69)$ \\
\hline & Absent & $63(80)$ & $11(17)$ & $52(83)$ \\
\hline \multirow{4}{*}{ Breslow $^{\#}$} & $\leq 1.0 \mathrm{~mm}$ & $24(30)$ & $2(8)$ & $22(92)$ \\
\hline & $1.01-2.0 \mathrm{~mm}$ & $23(29)$ & $3(13)$ & $20(87)$ \\
\hline & $2.01-4.0 \mathrm{~mm}$ & $6(8)$ & $3(50)$ & $3(50)$ \\
\hline & $>4.0 \mathrm{~mm}$ & $26(33)$ & $8(31)$ & $18(69)$ \\
\hline \multirow{2}{*}{ Sentinel lymph node } & Positive & $2(11)$ & $0(0)$ & $2(100)$ \\
\hline & Negative & $16(89)$ & $4(25)$ & $12(75)$ \\
\hline \multirow{4}{*}{ Follow-up } & Disease-free & $37(54)$ & $10(27)$ & $27(73)$ \\
\hline & Alive with metastases & $14(21)$ & $2(14)$ & $12(86)$ \\
\hline & Dead of melanoma & $14(21)$ & $2(14)$ & $12(86)$ \\
\hline & Lost & $3(4)$ & $2(67)$ & $1(33)$ \\
\hline
\end{tabular}

NY-ESO-1 (+): NY-ESO-1 positive; NY-ESO-1 (-): NY-ESO-1 negative; ${ }^{*} P<0.02$ for superficial spreading compared to other primary tumor types;

${ }^{\#} P$ value was not significant when Breslow index was analyzed as a categorical variable (groups); however, $P=0.017$ when Breslow index was analyzed as a continous variable.

TIL were graded as absent in $16 \%$ (11/68), nonbrisk in $72 \%$ $(49 / 68)$, and brisk in $12 \%(8 / 68)$ of the biopsies. NY-ESO-1 was expressed in $30 \%$ of tumors with nonbrisk infiltrates (14/47), 13\% with brisk TIL (1/8), and $8 \%$ with absent TIL (1/13), a difference not statistically significant (Figure 3). NYESO-1 patterns of expression in the melanoma (18\% scattered, $47 \%$ regional, and $35 \%$ complete), staining intensity, and percentage of positive tumor cells did not correlate with TIL grade.

We assessed TIL profile in 57 cases of vertical growth phase melanomas with either brisk or nonbrisk TIL, using immunohistochemical staining for CD3, CD8, and coexpression of CD8 and FoxP3 double stain. The mean number of CD3+ cells in brisk group was significantly higher than nonbrisk group $(P=0.0106)$. CD3+ T cell density and patterns of tumor infiltration correlated with NY-ESO-1 expression. $\mathrm{CD} 3+\mathrm{T}$ cells were more numerous in tumors negative for
NY-ESO-1 ( $P=0.017$, Figure 4$)$. Moreover, there was a correlation between large aggregates of $\mathrm{CD} 3+\mathrm{T}$ cells and absence of NY-ESO-1 ( $P=0.0001)$, whereas NY-ESO-1 positive tumors usually had isolated $(P=0.0019)$ and small clusters of CD3+ cells within the tumor $(P=0.0125$; Figure 5$)$.

NY-ESO-1 expression showed no correlation with the number of CD8+FoxP3- $\mathrm{T}$ cells or their distribution in isolated, small, or big clusters. Nor was there an association of NY-ESO-1 with CD8-FoxP3+ or CD8+FoxP3+ T cells (Figure 4).

\section{Discussion}

Given the dogma that cancer-testis antigens, such as NYESO-1, are associated only with cancer, any presence of NYESO-1 would indicate malignancy. This was confirmed by our and another study showing no expression in 20 and 19 nevi, 


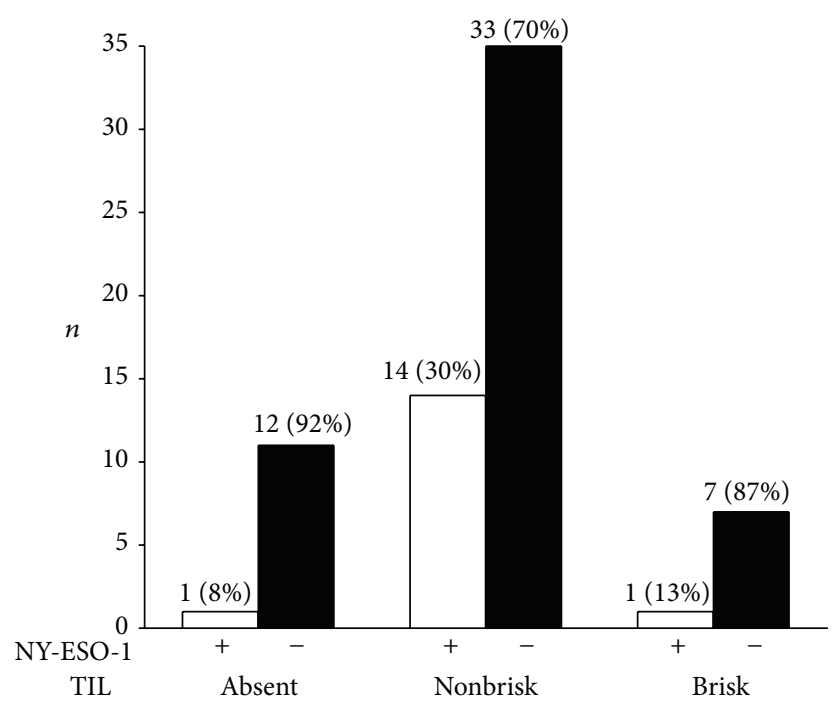

Figure 3: Tumor infiltrating lymphocytes (TIL) and NY-ESO-1 expression in primary cutaneous melanoma. NY-ESO-1 was associated with nonbrisk versus brisk and absent TIL $(P=0.07)$.

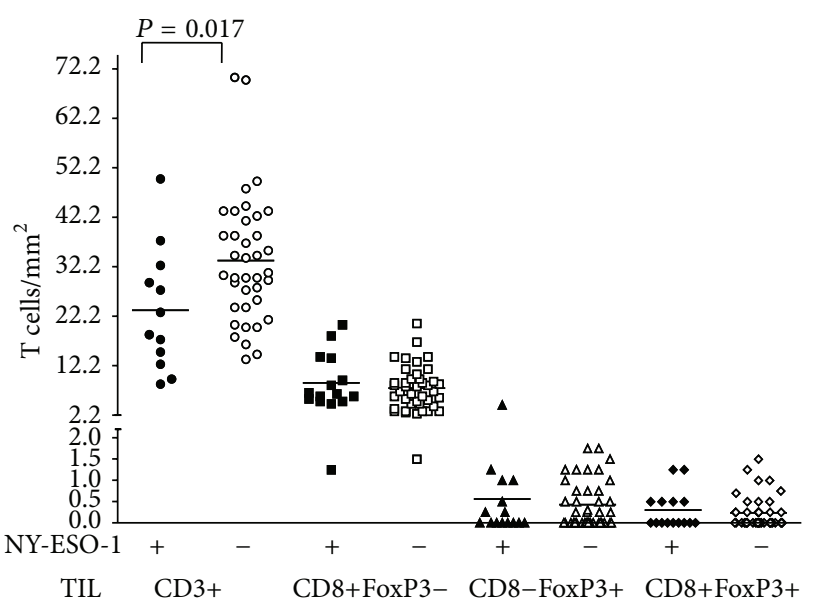

Figure 4: Number of CD3+, CD8+FOXP3-, CD8-FOXP3+, and $\mathrm{CD} 8+\mathrm{FOXP} 3+$ lymphocytes in NY-ESO-1 positive and negative primary cutaneous melanoma. Each geometric figure represents one patient. The horizontal bars represent the mean value.

respectively [37]. We observed NY-ESO-1 expression in 20\% (16/79) of invasive cutaneous melanomas, in agreement with the rate of 20 to $40 \%$ observed in other reports [28-31].

A recent study showed that $37 \%$ of 348 primary cutaneous melanomas were NY-ESO-1 positive by immunohistochemical analysis (IHC) [28]. Other authors assessing 586 melanoma samples (IHC) observed positivity of $46 \%$ for NYESO-1, including 251 primary melanomas and 335 metastases [29]. As an alternative, PCR testing done in 52 patients with melanoma showed that $33 \%$ of tumors were positive for NY-ESO-1 [30]. In another research, metastatic melanomas had a higher NY-ESO-1 expression of 32\% compared to $13 \%$ in primary cutaneous melanoma (IHC) [31]. The variability

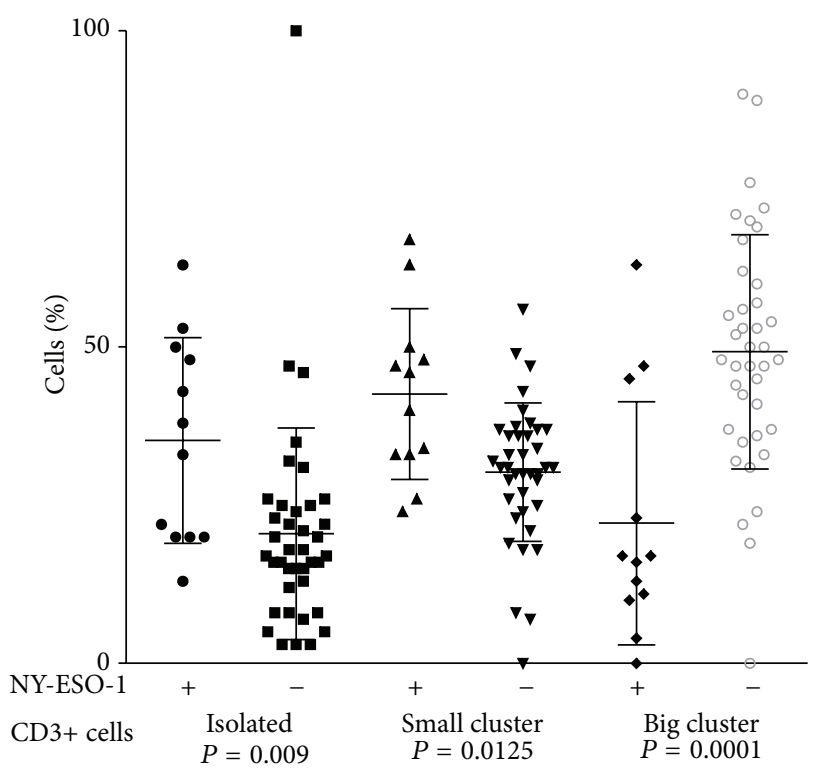

FIgURE 5: Arrangement of CD3+ cells in TIL of NY-ESO-1 positive and negative primary cutaneous melanoma. Each geometric figure represents the percentage of cells in one patient. The horizontal bars represent the mean value.

between these studies may be due to the stage of melanoma and the use of different laboratory techniques.

In the present study, NY-ESO-1 expression was associated with melanocytic tumor progression. Of 20 cases of benign nevi, 10 melanomas in situ, and 11 radial growth phase melanomas, only one melanoma in situ had detectable NYESO-1. On the other hand, $24 \%$ of vertical growth phase melanomas had detectable NY-ESO-1 protein (16/68).

We showed that NY-ESO-1 protein expression is associated with tumor thickness. Other researchers had similar findings. One study evaluating 251 cutaneous melanomas showed increased NY-ESO-1 in the intermediate group, between 1.1 and $4.0 \mathrm{~mm}$ of thickness, when compared with melanoma $<1.1 \mathrm{~mm}$ [29]. In another one, which analyzed 61 cutaneous melanomas, the NY-ESO-1 positive tumors had a median thickness of $4.7 \mathrm{~mm}$ versus $1.53 \mathrm{~mm}$ in the NY-ESO-1 negative group [31]. Recently, 321 patients with cutaneous melanoma also presented higher NY-ESO-1 antigen expression with increasing tumor thickness [28]. Our results and those previously published support the association between NY-ESO-1 protein expression and advanced melanomas, more invasive and thicker. However, a causal relationship cannot be established, as these findings come from retrospective cohort studies with biopsy done only once.

The expression of NY-ESO-1 protein in primary cutaneous melanoma is markedly heterogeneous. In our study, $33 \%$ of melanomas presented complete expression, regional expression in part of the tumor was observed in $47 \%$, and, less commonly, a diffusely scattered expression was seen in $18 \%$. These findings are somewhat different from those previously reported, wherein a diffuse pattern was the most common and focal (regional) distribution was the least common [29]. Another series reported yet different results, with $37 \%$ 
complete, 13\% regional, and 50\% scattered expression [31]. Therefore, the studies have consistently showed significant intertumoral heterogeneity in the expression of NY-ESO1 protein. We also observed significant variability in the percentage of tumor cells expressing NY-ESO-1 protein: $41 \%$ of the NY-ESO-1 $(+)$ melanomas $(n=7)$ expressed the protein in $2-20 \%$ of tumor cells, $18 \%(n=3)$ expressed the protein in $21-60 \%$ of tumor cells, and $41 \%(n=7)$ showed NY-ESO-1 protein in more than $60 \%$ of the tumor cells.

Despite the scientific evidence of an association between NY-ESO-1 expression and tumor thickness in primary cutaneous melanoma, an association with prognosis has not been confirmed. Cancer-testis antigen expression has been reported as an adverse prognostic indicator in a wide range of cancers, including neuroblastoma, ovarian cancer, breast cancer, and multiple myeloma [38-41]. NY-ESO-1 expression associated with poor prognosis in melanoma has been reported by some [29-31]. Nevertheless, our results and another report showed no association with overall survival [28]. We found no association of NY-ESO-1 expression with gender, age, race, skin phototype, tumor anatomic location, or sentinel lymph node status, findings that are consistent with a prior study [28].

As a potential immunogenic protein, it is important to know how NY-ESO-1 is related to the immune response and not just whether it is present or not. The tumor immunological environment is critical to the impact, if any, of NYESO-1 protein expression on prognosis. It is well established that the presence of TIL is associated with better prognosis in melanoma [42-45]. The present study included all grades of TIL (absent, nonbrisk, and brisk) but showed no statistically significant association between NY-ESO-1 and TIL grade. Another study has also reported a heterogeneous association of NY-ESO-1 expression and grade of TIL. Of 77 primary melanomas expressing NY-ESO-1, 57\% had absent TIL, 38\% nonbrisk TIL, and $5 \%$ brisk TIL, but the difference was not significant [28].

In order to further explore the immune environment, we assessed specific $\mathrm{T}$ cell subsets. Similar to the protective effect reported for TIL in general, CD3+ cells in particular have been associated with improved survival in cancer [46]. It is likely that the composition of the lymphocytic infiltrate is critical in this setting. We showed fewer CD3+ T cells in NY-ESO-1 (+) melanomas, and these cells were isolated or in small clusters, while, in the negative NY-ESO-1 tumors, the number of CD3+ cells was higher and the cells were arranged in large aggregates. Although NY-ESO-1 expression was not associated with the grade of TIL, it was associated with fewer infiltrating $\mathrm{CD} 3+\mathrm{T}$ cells, a puzzling finding not described before. It would be expected that NY-ESO-1 expression could induce and enhance an antitumor immune response.

Recent studies have also shown that CD8+ TIL are associated with better prognosis [46-48], and improved survival was associated with higher CD8/FoxP3 rates [46]. Using dual staining, we assessed CD8 and FoxP3 in relationship to NY-ESO-1 staining. While we observed fewer CD3+ cells associated with increased NY-ESO-1 expression, no association between NY-ESO-1 and CD8+FoxP3-, CD8-FoxP3+, or CD8+FoxP3+ cells in TIL was seen.
Clearly, the interplay between NY-ESO-1 and the immune response is very complex and large prospective cohorts investigating this interaction are need. Nevertheless, the association of NY-ESO-1 expression and the composition of the tumor lymphocytic infiltrate provide insights into the physiopathogenesis of immune response in primary melanoma and may drive future immunotherapeutic approaches [49].

\section{Conclusions}

NY-ESO-1 is a melanocytic tumor progression marker that is not present in benign nevi but is observed in approximately one-fifth of primary cutaneous melanomas, more commonly in thicker tumors and those with vertical growth phase. NY-ESO-1 protein expression displays marked heterogeneity, from $2 \%$ to $100 \%$ of the tumor cells, with varying expression patterns.

The present study is original in showing that NY-ESO-1 positive melanomas had fewer CD3+ TIL, and the T cells were most often dispersed as isolated cells or small clusters.

\section{Conflict of Interests}

The authors have no financial disclosure or conflict of interests.

\section{Authors' Contribution}

Mara Giavina-Bianchi, Cyro Festa-Neto, Mirian Nacagami Sotto, and Lyn M. Duncan were involved in the acquisition of the data. Mara Giavina-Bianchi, Pedro Giavina-Bianchi, Jorge Kalil, Cyro Festa-Neto, and Lyn M. Duncan contributed to the study concept and design, analysis, and interpretation of the data. Mirian Nacagami Sotto and Alona Muzikansky performed analysis and interpretation of the data. All authors carried out critical revision of the paper for this submission.

\section{Acknowledgments}

The authors acknowledge Ludwig Institute for Cancer Research, Brazil, for the donation of antibody E978 and also recognized the expert technical support of the Laboratorio Diagnostika, Brazil, for performing immunohistochemical stains and controls. They want to thank Patricia Della Pelle for $\mathrm{T}$ cell immunohistochemical staining. This study was supported by grants from FAPESP (Fundação de Amparo à Pesquisa do Estado de São Paulo), and CNPQ (Conselho Nacional de Pesquisa), Brazil, and the consultative funds of one of the authors (Lyn M. Duncan).

\section{References}

[1] B. K. Armstrong and A. Kricker, "Cutaneous melanoma," Cancer Surveys, vol. 19-20, pp. 219-240, 1994.

[2] E. Linos, S. M. Swetter, M. G. Cockburn, G. A. Colditz, and C. A. Clarke, "Increasing burden of melanoma in the United States," Journal of Investigative Dermatology, vol. 129, no. 7, pp. 16661674, 2009. 
[3] G. Pellacani, G. Lo Scocco, M. Vinceti et al., "Melanoma epidemic across the millennium: time trends of cutaneous melanoma in Emilia-Romagna (Italy) from 1997 to 2004," Journal of the European Academy of Dermatology and Venereology, vol. 22, no. 2, pp. 213-218, 2008.

[4] A. J. Stratigos, A. M. Forsea, R. J. T. van der Leest et al., "Euromelanoma: a dermatology-led European campaign against nonmelanoma skin cancer and cutaneous melanoma. Past, present and future," British Journal of Dermatology, vol. 167, supplement 2, pp. 99-104, 2012.

[5] H. B. Neuman, A. Patel, N. Ishill et al., "A single-institution validation of the AJCC staging system for stage IV melanoma," Annals of Surgical Oncology, vol. 15, no. 7, pp. 2034-2041, 2008.

[6] P. B. Chapman, A. Hauschild, C. Robert et al., "Improved survival with vemurafenib in melanoma with BRAF V600E mutation," The New England Journal of Medicine, vol. 364, no. 26, pp. 2507-2516, 2011.

[7] M. C. Fox, C. D. Lao, J. L. Schwartz, M. L. Frohm, C. K. Bichakjian, and T. M. Johnson, "Management options for metastatic melanoma in the era of novel therapies: a primer for the practicing dermatologist: part I: management of stage III disease," Journal of the American Academy of Dermatology, vol. 68, no. 1, pp. 1.e1-1.e12, 2013.

[8] F. S. Hodi, S. J. O’Day, D. F. McDermott et al., "Improved survival with ipilimumab in patients with metastatic melanoma," The New England Journal of Medicine, vol. 363, no. 8, pp. 711723,2010

[9] U. Keilholz, C. J. A. Punt, M. Gore et al., "Dacarbazine, cisplatin, and interferon-alfa-2b with or without interleukin-2 in metastatic melanoma: a randomized phase III trial (18951) of the European Organisation for Research and Treatment of Cancer Melanoma Group," Journal of Clinical Oncology, vol. 23, no. 27, pp. 6747-6755, 2005.

[10] C. Robert, L. Thomas, I. Bondarenko et al., "Ipilimumab plus dacarbazine for previously untreated metastatic melanoma," The New England Journal of Medicine, vol. 364, no. 26, pp. 25172526, 2011.

[11] A. Juretic, G. C. Spagnoli, E. Schultz-Thater, and B. Sarcevic, "Cancer/testis tumour-associated antigens: immunohistochemical detection with monoclonal antibodies," The Lancet Oncology, vol. 4, no. 2, pp. 104-109, 2003.

[12] A. J. G. Simpson, O. L. Caballero, A. Jungbluth, Y.-T. Chen, and L. J. Old, "Cancer/testis antigens, gametogenesis and cancer," Nature Reviews Cancer, vol. 5, no. 8, pp. 615-625, 2005.

[13] I. D. Davis, W. Chen, H. Jackson et al., "Recombinant NY-ESO-1 protein with ISCOMATRIX adjuvant induces broad integrated antibody and $\mathrm{CD}^{+}$and $\mathrm{CD}^{+} \mathrm{T}$ cell responses in humans," Proceedings of the National Academy of Sciences of the United States of America, vol. 101, no. 29, pp. 10697-10702, 2004.

[14] S. Gnjatic, E. Jäger, W. Chen et al., "CD8 ${ }^{+} \mathrm{T}$ cell responses against a dominant cryptic HLA-A2 epitope after NY-ESO-1 peptide immunization of cancer patients," Proceedings of the National Academy of Sciences of the United States of America, vol. 99, no. 18, pp. 11813-11818, 2002.

[15] E. Jäger, S. Gnjatic, Y. Nagata et al., "Induction of primary NYESO-1 immunity: CD8+ T lymphocyte and antibody responses in peptide-vaccinated patients with NY-ESO-1+ cancers," Proceedings of the National Academy of Sciences of the United States of America, vol. 97, no. 22, pp. 12198-12203, 2000.

[16] S. A. Rosenberg, "Shedding light on immunotherapy for cancer," The New England Journal of Medicine, vol. 350, no. 14, pp. 1461-1463, 2004.
[17] A. M. Scott and J. Cebon, "Clinical promise of tumour immunology," The Lancet, vol. 349, supplement 2, pp. SII19-SII22, 1997.

[18] S. Fujita, H. Wada, A. A. Jungbluth et al., "NY-ESO-1 expression and immunogenicity in esophageal cancer," Clinical Cancer Research, vol. 10, no. 19, pp. 6551-6558, 2004.

[19] J. Konishi, S. Toyooka, M. Aoe et al., "The relationship between NY-ESO-1 mRNA expression and clinicopathological features in non-small cell lung cancer," Oncology Reports, vol. 11, no. 5, pp. 1063-1067, 2004.

[20] T. Nakada, Y. Noguchi, S. Satoh et al., "NY-ESO-1 mRNA expression and immunogenicity in advanced prostate cancer," Cancer Immun, vol. 3, article 10, 2003.

[21] J.-R. Peng, H.-S. Chen, D.-C. Mou et al., "Expression of cancer/testis (CT) antigens in Chinese hepatocellular carcinoma and its correlation with clinical parameters," Cancer Letters, vol. 219, no. 2, pp. 223-232, 2005.

[22] Y. Wang, X. J. Wu, A. L. Zhao et al., "Cancer/testis antigen expression and autologous humoral immunity to NY-ESO-1 in gastric cancer," Cancer Immunity, vol. 4, p. 11, 2004.

[23] D. A. Chitale, A. A. Jungbluth, D. S. Marshall et al., "Expression of cancer-testis antigens in endometrial carcinomas using a tissue microarray," Modern Pathology, vol. 18, no. 1, pp. 119-126, 2005.

[24] J. A. Hemminger, A. Ewart Toland, T. J. Scharschmidt et al., "The cancer-testis antigen NY-ESO-1 is highly expressed in myxoid and round cell subset of liposarcomas," Modern Pathology, vol. 26, no. 2, pp. 282-288, 2013.

[25] T. Kurashige, Y. Noguchi, T. Saika et al., "NY-ESO-1 expression and immunogenicity associated with transitional cell carcinoma: correlation with tumor grade," Cancer Research, vol. 61, no. 12, pp. 4671-4674, 2001.

[26] K. Odunsi, A. A. Jungbluth, E. Stockert et al., "NY-ESO-1 and LAGE-1 cancer-testis antigens are potential targets for immunotherapy in epithelial ovarian cancer," Cancer Research, vol. 63, no. 18, pp. 6076-6083, 2003.

[27] H. A. Vaughae, S. Svobodova, D. MacGregor et al., "Immunohistochemical and molecular analysis of human melanomas for expression of the human cancer-testis antigens NY-ESO-1 and LAGE-1," Clinical Cancer Research, vol. 10, no. 24, pp. 83968404, 2004.

[28] S. Svobodová, J. Browning, D. MacGregor et al., "Cancertestis antigen expression in primary cutaneous melanoma has independent prognostic value comparable to that of Breslow thickness, ulceration and mitotic rate," European Journal of Cancer, vol. 47, no. 3, pp. 460-469, 2011.

[29] C. Barrow, J. Browning, D. MacGregor et al., "Tumor antigen expression in melanoma varies according to antigen and stage," Clinical Cancer Research, vol. 12, no. 3 I, pp. 764-771, 2006.

[30] J. S. Goydos, M. Patel, and W. Shih, "NY-ESO-1 and CTp11 expression may correlate with stage of progression in melanoma," Journal of Surgical Research, vol. 98, no. 2, pp. 76-80, 2001.

[31] E. F. Velazquez, A. A. Jungbluth, M. Yancovitz et al., "Expression of the cancer/testis antigen NY-ESO-1 in primary and metastatic malignant melanoma (MM) - correlation with prognostic factors," Cancer Immunity, vol. 7, article 11, 2007.

[32] T. Boon and L. J. Old, "Cancer tumor antigens," Current Opinion in Immunology, vol. 9, no. 5, pp. 681-683, 1997.

[33] B. Weide, H. Zelba, E. Derhovanessian et al., "Functional T cells targeting NY-ESO-1 or Melan-A are predictive for survival of 
patients with distant melanoma metastasis," Journal of Clinical Oncology, vol. 30, no. 15, pp. 1835-1841, 2012.

[34] A. Piris, A. C. Lobo, and L. M. Duncan, "Melanoma staging: where are we now?" Dermatologic Clinics, vol. 30, no. 4, pp. 581592, 2012.

[35] C. S. Kasper and M. D. Tharp, "Quantification of cutaneous mast cells using morphometric point counting and a conjugated avidin stain," Journal of the American Academy of Dermatology, vol. 16, no. 2 I, pp. 326-331, 1987.

[36] L. M. Duncan, L. A. Richards, and M. C. Mihm Jr., "Increased mast cell density in invasive melanoma," Journal of Cutaneous Pathology, vol. 25, no. 1, pp. 11-15, 1998.

[37] M. Lüftl, G. Schuler, and A. A. Jungbluth, "Melanoma or not? Cancer testis antigens may help," British Journal of Dermatology, vol. 151, no. 6, pp. 1213-1218, 2004.

[38] V. C. C. Andrade, A. L. Vettore, R. S. Felix et al., "Prognostic impact of cancer/testis antigen expression in advanced stage multiple myeloma patients," Cancer Immunity, vol. 8, article 2, 2008.

[39] S. Krüger, V. Ola, A. C. Feller, D. Fischer, and M. Friedrich, "Expression of cancer-testis antigen CT7 (MAGE-C1) in breast cancer: an immunohistochemical study with emphasis on prognostic utility," Pathology and Oncology Research, vol. 13, no. 2, pp. 91-96, 2007.

[40] E. Yakirevich, E. Sabo, O. Lavie, S. Mazareb, G. C. Spagnoli, and M. B. Resnick, "Expression of the MAGE-A4 and NY-ESO-1 Cancer-Testis Antigens in Serous Ovarian Neoplasms," Clinical Cancer Research, vol. 9, no. 17, pp. 6453-6460, 2003.

[41] A. Oberthuer, B. Hero, R. Spitz, F. Berthold, and M. Fischer, "The tumor-associated antigen PRAME is universally expressed in high-stage neuroblastoma and associated with poor outcome," Clinical Cancer Research, vol. 10, no. 13, pp. 4307-4313, 2004.

[42] F. Azimi, R. A. Scolyer, P. Rumcheva et al., “Tumor-infiltrating lymphocyte grade is an independent predictor of sentinel lymph node status and survival in patients with cutaneous melanoma," Journal of Clinical Oncology, vol. 30, no. 21, pp. 2678-2683, 2012.

[43] W. H. Clark Jr., D. E. Elder, D. Guerry IV et al., "Model predicting survival in stage I melanoma based on tumor progression," Journal of the National Cancer Institute, vol. 81, no. 24, pp. 1893-1904, 1989.

[44] C. G. Clemente, M. C. Mihm Jr., R. Bufalino, S. Zurrida, P. Collini, and N. Cascinelli, "Prognostic value of tumor infiltrating lymphocytes in the vertical growth phase of primary cutaneous melanoma," Cancer, vol. 77, no. 7, pp. 1303-1310, 1996.

[45] N. E. Thomas, K. J. Busam, L. From et al., "Tumor-infiltrating lymphocyte grade in primary melanomas is independently associated with melanoma-specific survival in the populationbased genes, environment and melanoma study," Journal of Clinical Oncology, vol. 31, no. 33, pp. 4252-4259, 2013.

[46] M. J. M. Gooden, G. H. de Bock, N. Leffers, T. Daemen, and H. W. Nijman, "The prognostic influence of tumour-infiltrating lymphocytes in cancer: a systematic review with meta-analysis," British Journal of Cancer, vol. 105, no. 1, pp. 93-103, 2011.

[47] I. S. van Houdt, B. J. R. Sluijter, L. M. Moesbergen et al., "Favorable outcome in clinically stage II melanoma patients is associated with the presence of activated tumor infiltrating Tlymphocytes and preserved MHC class I antigen expression," International Journal of Cancer, vol. 123, no. 3, pp. 609-615, 2008.

[48] F. Piras, R. Colombari, L. Minerba et al., "The predictive value of CD8, CD4, CD68, and human leukocyte antigen-D-related cells in the prognosis of cutaneous malignant melanoma with vertical growth phase," Cancer, vol. 104, no. 6, pp. 1246-1254, 2005.

[49] K. Odunsi, J. Matsuzaki, J. Karbach et al., "Efficacy of vaccination with recombinant vaccinia and fowlpox vectors expressing NY-ESO-1 antigen in ovarian cancer and melanoma patients," Proceedings of the National Academy of Sciences of the United States of America, vol. 109, no. 15, pp. 5797-5802, 2012. 


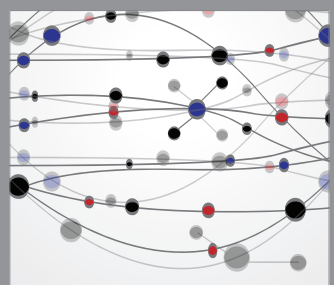

The Scientific World Journal
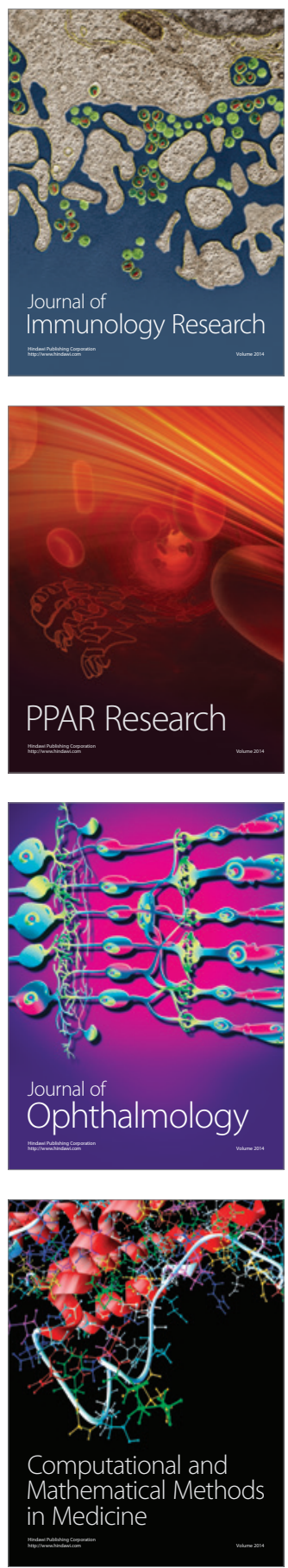

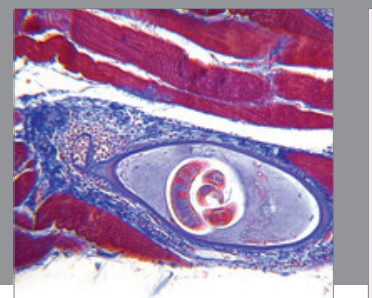

Gastroenterology

Research and Practice
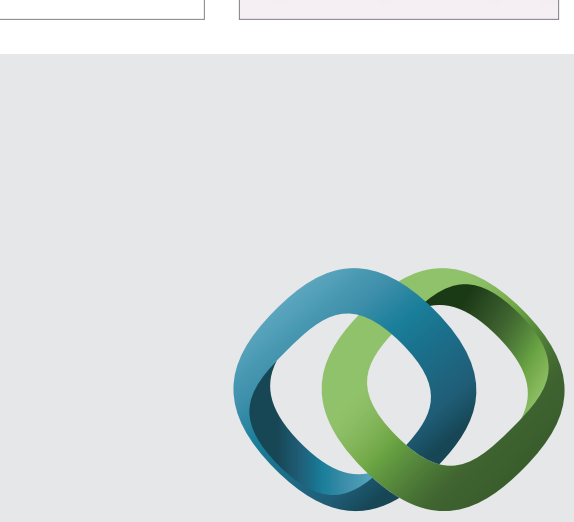

\section{Hindawi}

Submit your manuscripts at

http://www.hindawi.com
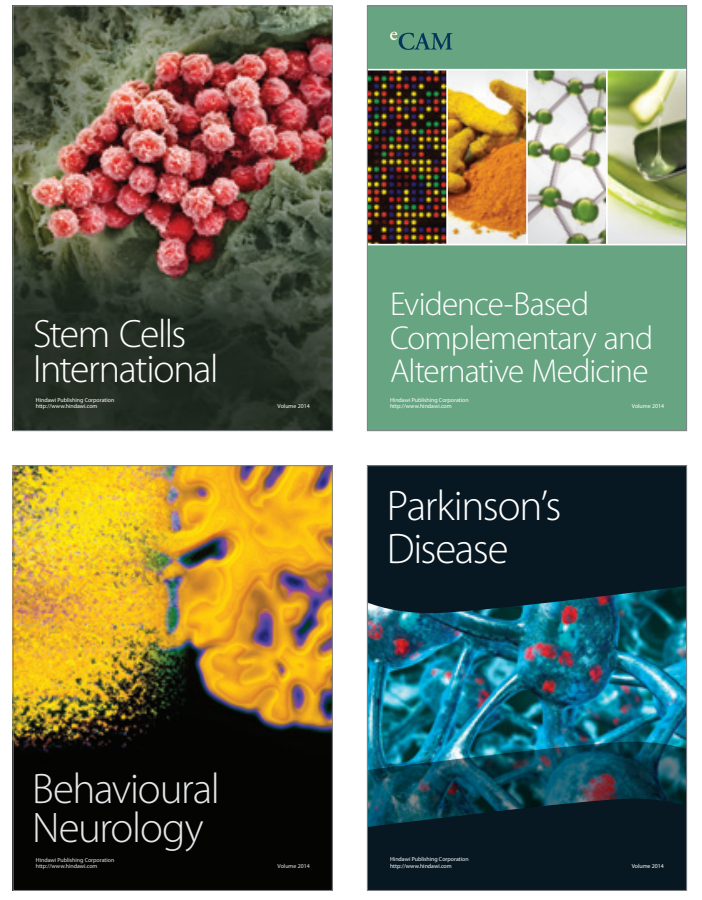
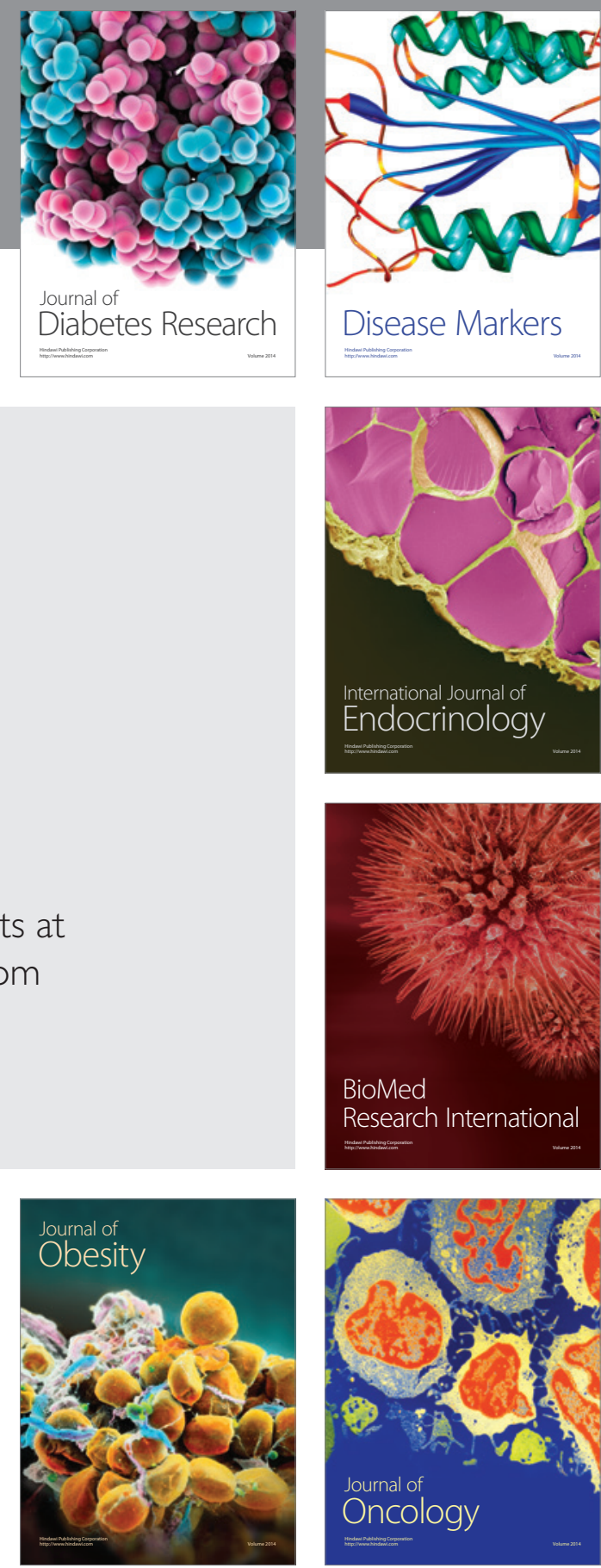

Disease Markers
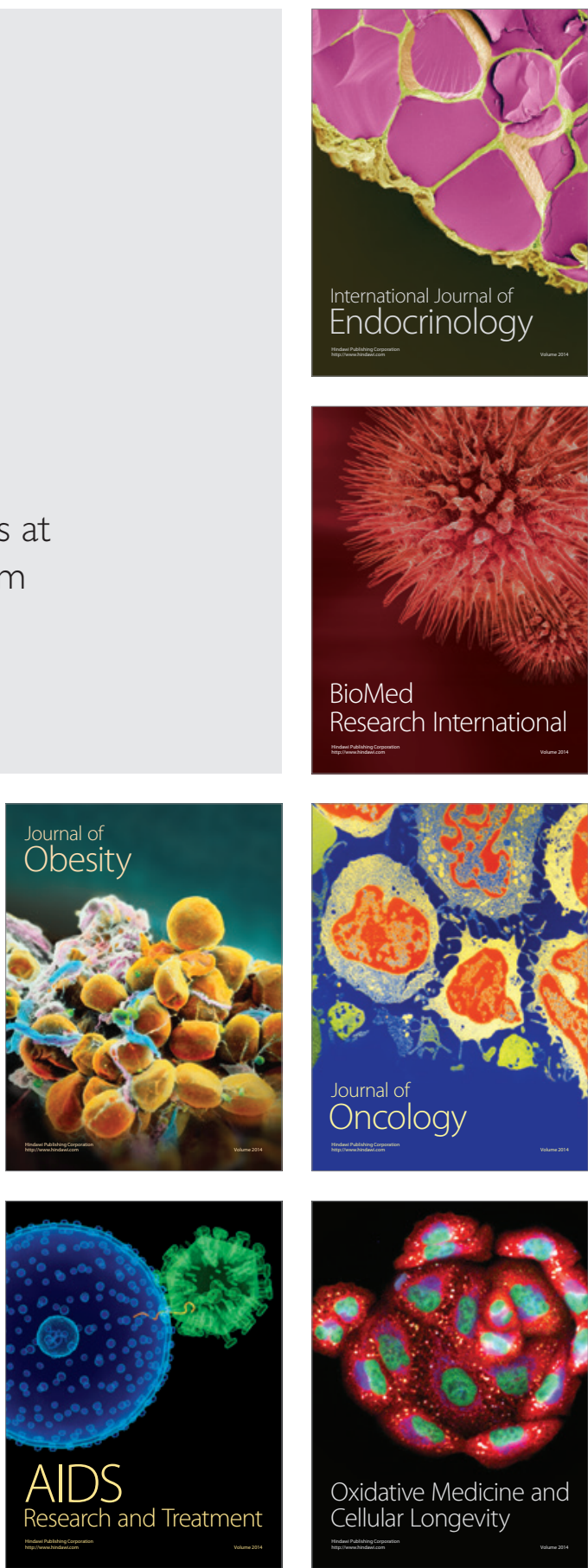